Chapter 2

\title{
The Cell-Multilayered Periosteal Sheet - A Promising Osteogenic and Osteoinductive Grafting Material
}

\author{
Tomoyuki Kawase, Kazuhiro Okuda, \\ Masaki Nagata and Hiromasa Yoshie \\ Additional information is available at the end of the chapter \\ http://dx.doi.org/10.5772/58902
}

\section{Introduction}

The present is an exciting time in the field of regenerative biology in dental medicine. Many basic and clinical studies have been performed to improve periodontal therapy and rehabilitation therapy for morphological and functional recovery of the maxillofacial bones. Many materials, e.g., hydroxyapatite, GTR membrane, enamel matrix derivative, and platelet-rich plasma, have been applied to osseous defects to achieve significant outcomes. In contrast, cell therapy, as an advanced tissue-engineering technology, using bone marrow stem cells, periosteal cells, or periodontal ligament cells have just been started in several university hospitals and institutes in Japan.

The periosteum has been recognized as a promising source of immature osteogenic progenitor cells. Osteogenic cells are important components as a promoter of bone regenerative therapy because their bioactivities are crucial to reconstituting an active site of controlled bone formation. We demonstrated that implantation of cultured periosteal sheets into nude mice could induce the formation of bone-like tissue [Kawase et al., 2009]. In addition, we established regenerative surgery with autologous cultured periosteal sheets in combination with plateletrich plasma (PRP) and hydroxyapatite (HA) in periodontitis patients (Fig. 1) [Yamamiya et al., 2008, Okuda et al.,2013]. Furthermore, we reported remarkable bone remodeling in reduced alveolar bone from autologous bone grafting with cultured periosteal sheets [Nagata et al., 2012].

In this chapter, we will introduce the characteristics of periosteal sheets and clinical outcome by cell-based therapy in patients with periodontitis and alveolar jaw reduction. In addition, future perspectives on regenerative therapy will be described. 


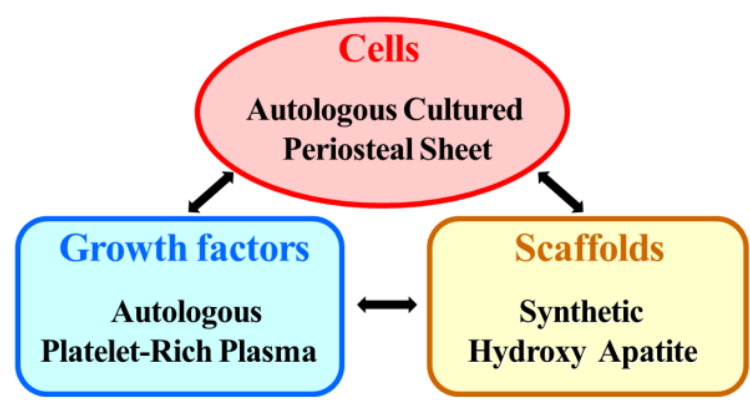

Figure 1. Cultured periosteal sheet application to infrabony defects in patients with periodontitis

\section{Biological characteristics of the cultured periosteal sheet}

Our periosteal sheets are prepared using static explant cultures of small pieces of alveolar periosteum tissue. The number of cells can be expanded at least by 1000 times by the end of the 6-week preparation, and the resulting periosteal sheet is mechanically tough enough to be picked up by forceps [Kawase et al., 2009]. In our experience preparing more than 2000 periosteal sheets, we confirmed that the growth is not significantly dependent either on age or sex. The successful preparation is rather dependent on the cell density in excised periosteum tissue segments. It is also important to handle the excised periosteum tissue in a timely manner, especially in the initial preparation of the "cut and paste" onto culture dishes because this procedure includes a time-sensitive semi-drying process for facilitating tissue segment attachment onto the plastic surface.

We developed this grafting material as a substitute for crushed autologous bone, a gold standard osteogenic grafting material. Therefore, the periosteal sheets are expected to express osteogenic and osteoinductive capabilities besides (or rather than) the osteoconductive capability displayed by most recent bone filler made of bioactive ceramics.

In our basic studies using in vitro cell culture systems and in vivo animal implantation systems, we have demonstrated several remarkable characteristics of the periosteal sheet, which are summarized below:

Tissue-like thickness: The periosteal sheet displays a unique structure that is composed of cell-multilayers and abundantly deposited extracellular matrices (ECM).

Osteogenicity: Periosteal cells within the periosteal sheet differentiate to osteogenic cells slowly but spontaneously with time of expansion using a conventional medium.

Osteoinduction: The periosteal sheet produces the major growth factors involved in bone metabolism. 


\subsection{Tissue-like thickness}

Because of the cell-multilayered structure, as shown in Figure 2 (Left), the periosteal sheet is substantially thicker than a monolayer cell sheet [Kawase et al., 2009]. In addition, the thickness of the periosteal sheet can be enhanced by many types of commercially available stem-cell culture media [Uematsu et al., 2013a, 2013b] (Fig. 2 Right). However, intact periosteum tissue is hardly excised for expansion culture, and the native periosteum-like structure, dual-layers composed of the cambium and fibrous layers, could not be newly formed or regenerated in vitro under our static culture expansion conditions.
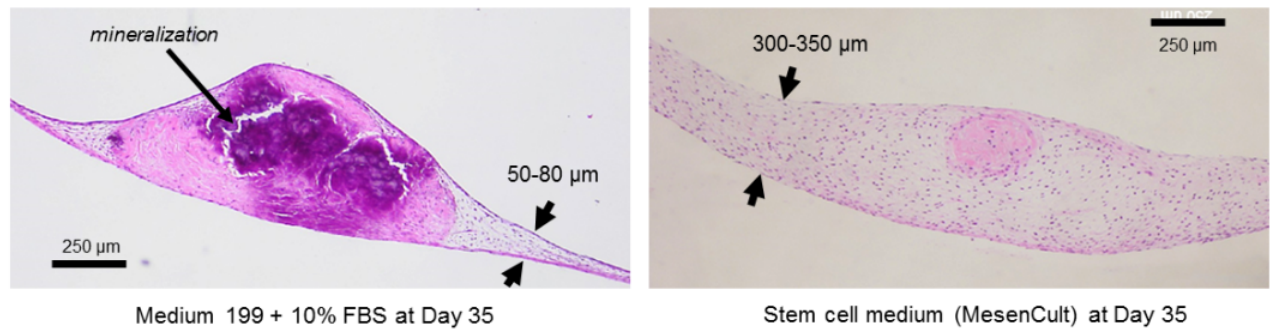

Figure 2. Effects of culture media on the morphology of periosteal sheets.

Periosteal sheets were expanded with the conventional growth medium (Medium 199 supplemented with 10\% FBS) or a commercial stem-cell medium (MesenCult) for 35 days. Saggital paraffin sections were stained with hematoxylin \& eosin.

Interestingly, as far as we could attempt and achieve, similar cell-multilayered structures can barely be formed by similar expansion cultures of other connective tissues, such as epidermal and periodontal ligament tissue [Kawase et al., Unpublished observations]. It is also impossible to form similar structures using dispersed single periosteal cell populations in conventional two-dimensional (2D) culture systems. However, using appropriate scaffolding materials, we developed a 3D culture system for preparation of similar periosteal sheets [Kawase et al., 2014].

\begin{tabular}{cccc}
\hline Material & Region & Stiffness & $\mathbf{n}$ \\
\hline Periosteal sheet & central & $5.59 \pm 3.24 \mathrm{kPa}$ & 76 \\
\hline Periosteal sheet & peripheral & $4.35 \pm 2.69 \mathrm{kPa}$ & 63 \\
\hline $\begin{array}{c}\text { Dispersed periosteal cells } \\
\text { Dispersed periosteal cells } \\
\text { (living) }\end{array}$ & $14.3 \pm 9.42 \mathrm{kPa}$ & 118 \\
\hline Tissue culture polystyrene & $9.04 \pm 9.20 \mathrm{kPa}$ & 54 \\
\hline
\end{tabular}

Stiffness was evaluated by the nanoindentation technique using an atomic force microscopy.

Table 1. The apparent stiffness of periosteal sheets. 
On the other hand, from a biomechanical point of view, ECM-enriched structures function as a soft scaffolding material for proliferating periosteal cells. As shown in Table 1, the stiffness of a conventional plastic dish is several GPa, whereas that of a light-fixed periosteal sheet is 4-6 kPa [Horimizu et al., 2013]. Because stiffer materials are in general more suitable for cell mitosis and proliferation [Vincent et al., 2013], it can be predicted that conventional culture dishes provide more preferable places for periosteal cells to proliferate. This is most likely a major reason why dispersed periosteal cells grow faster than cells contained in a periosteal sheet. It has also been reported that the optical stiffness for the osteoblastic differentiation is $25-40 \mathrm{kPa}$, and softer material surfaces maintain cells in the osteoblast lineage at immature stages [Engler et al., 2006]. Therefore, it is presumable that a thicker periosteal sheet could be suitable for the expansion of immature osteoprogenitor cells without inducing the osteoblastic differentiation.

\subsection{Osteogenicity}

As a promising osteogenic grafting material, a periosteal sheet should produce mineral deposits in vitro and form bone-like tissue in vivo. In conventional growth medium, Medium 199 supplemented with 10\% fetal bovine serum (FBS), periosteal cells gradually and increasingly express alkaline phosphatase (ALP) with expansion time [Kawase et al., 2009]. The addition of chemical agents involved in osteoblastic differentiation, such as dexamethasone, $\beta$-glycerophosphate, and ascorbate, immediately increases ALP activity and simultaneously mineral deposit formation [Kawase et al., 2009, 2010, 2011].

When implanted into the subcutaneous tissue of nude mice, these periosteal sheets, regardless of their differentiation stages, are capable of forming osteoid and bone-like tissue more potently than dispersed periosteal cells combined with porous hydroxyapatite blocks [Kawase et al., 2009, Uematsu et al., 2013a]. In addition, several periosteal cells, especially those embedded in dense collagen matrices, become osteocyte-like cells. These data suggest the "osteogenicity" of the periosteal sheet.

\subsection{Osteoinduction}

We have detected major growth factors, such as several interleukins, FGF-2 (fibroblast growth factor-2), MCP-1 (monocyte chemotactic protein-1), TPO (thrombopoeitin), IGFBP-2 (insulinlike growth factor binding protein-2), GRO (growth-related oncogene) and VEGF (vascular endothelial growth factor) in conditioned media of the periosteal sheet [Kawase et al., 2009, Kobayashi et al., Manuscript in submission]. The osteogenic induction using chemical agents drastically changed the profile of cytokine release, as shown in Table 2.

Following implantation of periosteal sheets into the subcutaneous tissue of nude mice, global DNA microarray analyses demonstrated that the periosteal sheet influences the surrounding host tissue and induces osteoclastic phenotypes (Table 3). We also observed that the osteoblastic phenotypes could be induced by the implanted periosteal sheet. In addition, the osteoinduced periosteal sheet is potently capable of inducing angiogenesis and osteoclastic formation. Therefore, these data suggest "osteoinduction" by the periosteal sheet. 


\begin{tabular}{ll}
\hline \multicolumn{1}{c}{ Changes } & Growth factors \\
\hline control $>$ differentiated & IL-6 MCP-1, TPO \\
\hline control < differentiated & GRO- $a$, IL-8, IL-13, IGFBP-2 \\
\hline moderate & GRO, IL-5, IL-7, IL-10, MCP-3 \\
\hline substantial & \\
\hline $\begin{array}{l}\text { Growth factors released by the control and the osteoinduced periosteal sheets to the low-serum culture medium were } \\
\text { semi-quantitatively evaluated using an antibody array. }\end{array}$
\end{tabular}

Table 2. Effects of the osteoinduction on the growth factor production by the periosteal sheet.

\begin{tabular}{|c|c|}
\hline Phenotype & Genes \\
\hline \multicolumn{2}{|l|}{ Upregulation (> twofolds) } \\
\hline Osteoblast & RUNX2, SatB2, Osteoprotegerin, BMP1, TGF $\beta 1$, integrin a5 $\beta 1$, ICAM1 \\
\hline Osteoclast & TRAP, Cathepsin K, Osteoclast-like cell cDNA \\
\hline Blood vessel & Tie1 \\
\hline \multicolumn{2}{|c|}{ Downregulation (> twofolds) } \\
\hline Stem cell & SOX2 \\
\hline Chondrocyte & SOX9, COL2 \\
\hline Osteoblast & MSX2, ALP, BMP4, Bone gla protein, IGF2 \\
\hline Osteoclast & CSF1 \\
\hline \multicolumn{2}{|c|}{$\begin{array}{l}\text { The human periosteal sheets, which were implanted into the subcutaneous tissue of nude mice, were retrieved along } \\
\text { with the surrounding mouse tissue. Mouse-specific mRNAs expressed in the surrounding tissue were evaluated by an } \\
\text { Agilent Mouse Genome }(4 \times 44 K) \text { microarray. }\end{array}$} \\
\hline
\end{tabular}

Table 3. Global DNA microarray analyses of gene expression in the surrounding mouse connective tissue.

\subsection{Conclusion}

We should again emphasize that the periosteal sheet is characterized by its unique structure. Dispersed periosteal cells and other cells in the osteoblast lineage expanded in 2D culture systems are capable of producing growth factors involved in bone metabolism and forming mineral deposits as well as the periosteal sheet. However, these cells require appropriate scaffolding materials for implantation. In addition, a 3D culture system without using appropriate scaffolding materials, such as accumulation of cell sheets and large-scale spheroid 
formations, typically induces cell necrosis in the central regions. Abundant ECM functions as a scaffolding material and avoids the increase in cell density, preventing inevitable necrosis.

\section{Application of cultured periosteal sheet for periodontal regeneration}

To regenerate periodontal tissue destroyed by chronic periodontitis, human autologous cultured periosteal sheets in combination with platelet-rich plasma (PRP) [Okuda et al., 2003] and hydroxyapatite (HA) granules, were clinically applied. The periosteum specimens were dissected from each patient and were incubated for approximately six weeks until the sheets were formed. Prior to placement of the cultured periosteal sheets, osseous defects were thoroughly debrided and filled with HA granules in combination with PRP. Standardized clinical and radiographic measurements were performed during follow-up examinations at one and five years. Clinical attachment level (CAL) and radiographic infrabony defect depth (IBD) were determined as the primary and secondary end-points.

The one-year results of this treatment indicate favorable clinical results, when compared to baseline or when compared to the control group (PRP with HA). A five-year follow-up evaluation of 22 selected patients after cultured periosteal sheets treatment indicated the treated infrabony defects remained clinically stable and were well maintained. Radiographically, there was increased osseous radiopacity and bone trabeculation, suggesting further bone maturation.

A factor likely contributing to these favorable clinical results is the presence of osteogenic cells in the cultured periosteal sheets, which provided greater regeneration potential.

\subsection{Case presentation}

In this case report, clinical and radiographic evaluation of the clinically representative case \#22 of a cultured periosteal sheet in combination with a PRP and HA granule mixture surgically treated site is presented in Figure 3.

A 56-year-old Japanese female presented with clinical and radiographic (Fig. 3A) evidence of chronic periodontitis and bone loss on the mesial surface of the mandibular left first molar. At baseline, the PD was $6 \mathrm{~mm}$, and the CAL was $6 \mathrm{~mm}$. Upon surgical exposure, a 5-mm occlusalapical by 5 -mm mesio-distal three-walled infrabony osseous defect was revealed on the mesial surface of the molar (Fig. 3B). After debridement and thorough root planing, a PRP and HA granule complex was placed into the osseous infrabony defect (Fig. 3C) and overlaid with a cultured periosteal sheet (Fig. 3D).

At 1-year post-surgery, the PD and CAL were $3 \mathrm{~mm}$ and $5 \mathrm{~mm}$, respectively, representing a 1$\mathrm{mm}$ gain in CAL from baseline, and this favorable clinical response in the decrease of PD and gain in CAL was maintained at 5 years (Fig. 3E). Increased radiopacity was demonstrated on the mesial surface of the molar at 1 year and 5 years (Fig. 3F) [Okuda et al., 2009, 2013]. 

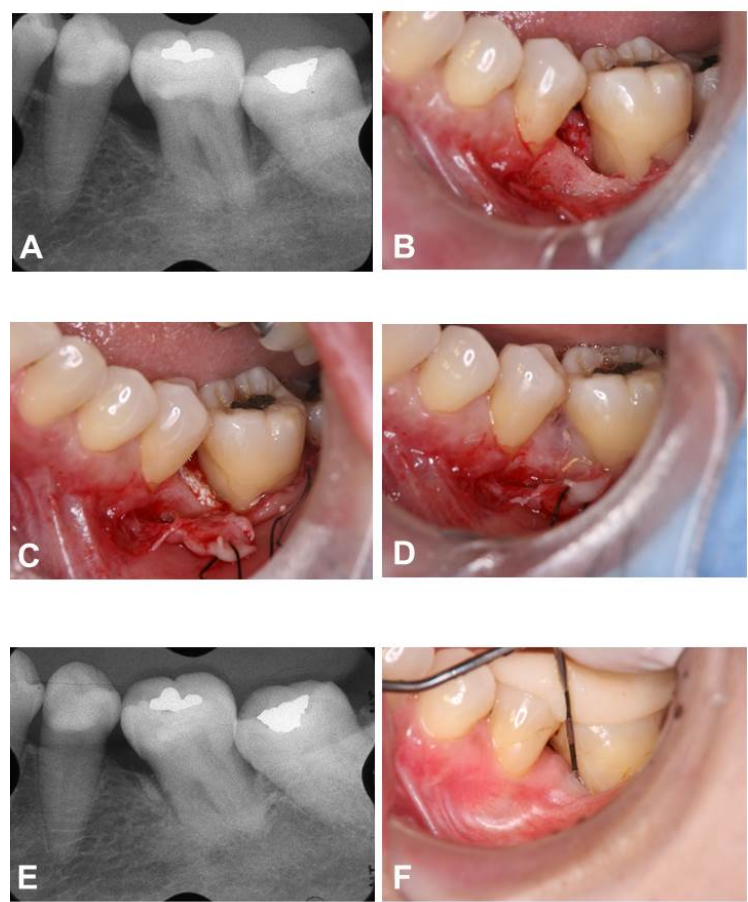

Figure 3. Clinical and radiographic appearance during follow-up examinations at baseline and five years. A: Surgical area before surgery with IBD depicting $5 \mathrm{~mm}$ of bone loss, B: Reflection and debridement of the area revealed a threewalled osseous defect, C: Grafting with PRP+HA granules, D: The surgical site following placement of the cultured periosteal sheet covering the PRP+HA granules, E: Clinical view at 5-year follow-up, F: Radiographs at 5 years showing that the cortical bony architecture and periodontal ligament space appear normal.

\subsection{Case-controlled clinical trial}

Thirty interproximal infrabony osseous defects in 30 healthy, non-smoking subjects diagnosed with chronic periodontitis were included in this study with one infrabony defect with PD $\geq 6$ $\mathrm{mm}, \mathrm{CAL} \geq 6 \mathrm{~mm}$, and an osseous defect depth estimated to be $\geq 3 \mathrm{~mm}$ when measured radiographically. The subjects were randomly assigned to a test group (cultured periosteal sheets combined with PRP and HA) or a control group (PRP with HA). Clinical and radiographic measurements were performed at baseline and the 1-year post-surgical evaluation. Compared to baseline, the 1-year results indicated that both treatment modalities resulted in significant changes $(P<0.01)$ in the probing depth, clinical attachment level, and radiographic infrabony defect depth. Compared to the control group, the test group exhibited a significantly more favorable change in clinical attachment $(4.1 \pm 1.2 \mathrm{~mm}$ versus $5.3 \pm 1.5 \mathrm{~mm} ; P<0.05)$ and radiographic infrabony defect $(0.4 \pm 0.9 \mathrm{~mm}$ versus $1.7 \pm 1.2 \mathrm{~mm} ; P<0.01)$, as shown in Table 4 [Okuda et al., 2005, Yamamiya et al., 2008]. 


\begin{tabular}{llll}
\hline Clinical and Radiographic Index & & & \\
\hline and Treatment Group & Baseline & 1-year & $P$ value $^{*}$ \\
\hline PD (mm) & & & \\
\hline Periosteal sheet + PRP + HA (Test) & $7.7 \pm 1.1$ & $2.9 \pm 0.4$ & $P<0.01$ \\
\hline PRP + HA (Control) & $7.6 \pm 1.1$ & $3.3 \pm 1.0$ & NS \\
\hline$P$ value ${ }^{\dagger}$ & NS & & $P<0.01$ \\
\hline CAL (mm) & & $4.1 \pm 1.2$ & $P<0.01$ \\
\hline Periosteal sheet + PRP + HA & $8.1 \pm 1.2$ & $5.3 \pm 1.5$ & \\
\hline PRP + HA & $8.0 \pm 1.3$ & $P<0.05$ & $P<0.01$ \\
\hline$P$ value & NS & & $P<0.01$ \\
\hline IBD (mm) & & $0.4 \pm 0.9$ & $1.7 \pm 1.2$ \\
\hline Periosteal sheet + PRP + HA & $5.3 \pm 1.1$ & $P<0.01$ & \\
\hline PRP + HA & $4.9 \pm 1.4$ & NS & \\
\hline$P$ value & & & \\
\hline
\end{tabular}

Means $\pm S D$ ( $n=15$ subjects in each treatment group). SD=standard deviation. NS=not significant, $P>0.05, *$ The significance level between baseline and 1-year, + Statistical significance level between the study groups at baseline or 1 year.

Table 4. Mean clinical and radiographic measurements at baseline and 1 year for the test (periosteal sheet+PRP+HA) and control (PRP+HA) study groups.

\subsection{Five-year follow-up study}

Twenty-two patients who had received surgery using cultured periosteal sheets were included in the study for statistical analysis comparing the 1-year results and the 5-year follow-up results. Significant differences between baseline and the 1-and 5-year data were evaluated by means of the Wilcoxon signed-rank matched-pair test, taking into account the paired nature of the observations. The mean PD, CAL and IBD scores at baseline are as follows: PD, $7.6 \pm 1.4$ $\mathrm{mm}$; CAL, $8.0 \pm 1.4 \mathrm{~mm}$ and IBD, $4.6 \pm 1.4 \mathrm{~mm}$ (Table 5).

The 1-year results after treatment indicated the mean PD, CAL and IBD values were improved significantly as compared with baseline (PD: $2.9 \pm 0.4 \mathrm{~mm}$ at 1-year versus $7.6 \pm 1.4 \mathrm{~mm}$ at baseline, $P<0.01$; CAL: $4.8 \pm 1.4 \mathrm{~mm}$ at 1 -year versus $8.0 \pm 1.4 \mathrm{~mm}$ at baseline, $P<0.01$; IBD: $1.4 \pm 1.1 \mathrm{~mm}$ at 1 -year versus $4.6 \pm 1.0 \mathrm{~mm}$ at baseline, $P<0.01$ ).

At the 5-year follow-up, as compared to the 1-year evaluation period, the mean PD and CAL were stable (PD: $2.9 \pm 0.3 \mathrm{~mm}$ at 5-years vs. $2.9 \pm 0.4 \mathrm{~mm}$ at 1 year, NS, CAL: $4.6 \pm 1.2 \mathrm{~mm}$ at 5year vs. $4.8 \pm 1.4 \mathrm{~mm}$ at 1-year; NS), and IBD was improved significantly when compared to the 1-year result $(0.2 \pm 0.5 \mathrm{~mm}$ at 5 -years vs. $1.4 \pm 1.1 \mathrm{~mm}$ at 1 -year, $P<0.01)$. It is also noteworthy that there were no infectious episodes and no other adverse complications associated with treatment over the five-year time period of this study [Okuda et al., 2013]. 


\begin{tabular}{|c|c|c|c|c|c|c|}
\hline & Baseline & 1-year & 5-years & & $p$ value & \\
\hline & & & & 1 vs $B L^{*}$ & $5 \mathrm{vs} \mathrm{BL}^{\dagger}$ & 5 vs $1^{\neq}$ \\
\hline \multicolumn{7}{|c|}{ Clinical Parameter } \\
\hline PD & $7.6 \pm 1.4$ & $2.9 \pm 0.4$ & $2.9 \pm 0.3$ & $P<0.01$ & $P<0.01$ & NS \\
\hline CAL & $8.0 \pm 1.4$ & $4.8 \pm 1.4$ & $4.6 \pm 1.2$ & $P<0.01$ & $P<0.01$ & NS \\
\hline IBD & $4.6 \pm 1.0$ & $1.4 \pm 1.1$ & $0.2 \pm 0.5$ & $P<0.01$ & $P<0.01$ & $P<0.01$ \\
\hline
\end{tabular}

Means $\pm S D$ ( $n=22$ subjects). SD=standard deviation, NS=not significant, $P>0.05, * 1$ year compared with baseline, +5 year compared with baseline, $\neq 5$ year compared with 1 year

Table 5. Mean clinical and radiographical data at each treated site at baseline and at the 1-and 5-year follow-up periods.

\subsection{Conclusion}

Compared to PRP with HA, treatment with a combination of cultured periosteal sheets, PRP, and HA led to a significantly more favorable clinical improvement in infrabony periodontal defects at 1-year post-surgery. However, the added benefits of cultured periosteal sheets had statistical and clinical significance. Once a successful clinical treatment has been achieved with a combination of cultured periosteal sheets, $\mathrm{PRP}$, and HA granules, the results are maintainable for 5 years. In addition to the favorable long-term clinical results for treating periodontal osseous defects, the long-term safety of the cultured periosteal sheets in periodontal therapy was established.

\section{Application of cultured autogenous periosteal cell sheets to alveolar bone regeneration}

Diseases affecting the maxillofacial region often cause defects in alveolar or gnathic bone. The maxillofacial bones are an important determinant of facial features and play a pivotal role in mastication and articulation. Thus, rehabilitation therapy helps morphological and functional recovery of the maxillofacial bones is an important component of the strategies required after the diseases have been treated. Autogenous bone grafting is the most realistic and effective method for regenerating bone tissue in defect sites and is therefore widely used. However, the bone harvesting is accompanied by unavoidable donor site morbidity such as pain and increased risk of infection.

For these types of reasons, we employed the tissue-engineering approach aided by the administration of cells derived from autogenous periosteum, which provided osteoblasts responsible for bone formation and also can promote the recruitment of cells responsible for bone resorption [Nagata et al., 2012, Uematsu et al., 2013a]. Patients scheduled to undergo alveolar bone augmentation prior to dental implant placement were subjected to clinical study of alveolar bone engineering with administration of cultured autogenous periosteal cell (CAPC) sheet. 


\subsection{CAPCs promoted good bone regeneration and reduced the amounts of bone required for harvesting}

CAPCs grafting with autologous bone was performed for alveolar ridge augmentation and for maxillary sinus lift (Fig. 4). The bone was harvested from the anterior region of the mandibular ramus in the majority of cases and from the iliac crest in one case. Bone regeneration after CAPCs grafting with autogenous bone was generally satisfactory, and the predictability of alveolar bone augmentation was high even in large recipient sites. Use of CAPCs resulted in alveolar ridge augmentation with satisfactory morphology and long-term stable bone volume in these advanced cases of atrophy. Furthermore, graft materials containing less than $40 \%$ of the autogenous bone particle also demonstrated good bone formation, and it was found that using CAPCs was beneficial for the satisfactory formation and long-term maintenance of regenerated bone. In other words, use of CAPCs can reduce the amount of autogenous bone required, which reduced donor site morbidity during bone harvesting.

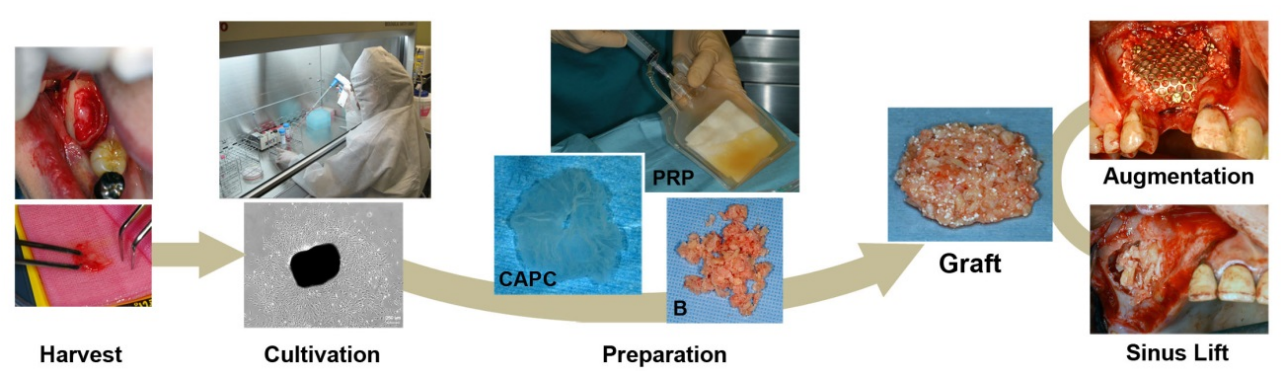

Figure 4. Procedure of autogenous bone grafting with cultured autogenous periosteal cells (CAPCs).

\subsection{Recruitment of osteoblasts and osteoclasts to regenerating bone tissue}

The histological images of biopsy specimens recovered from the recipient sites at 4 months after CAPCs grafting with particulated bone exhibit newly formed bone in spaces between the cortical bone particles in graft material (Fig. 5). Layers of cells strongly immunopositive for ALP activity were present on the surface of the newly formed bone. Recruitment of TRAPpositive cells was confirmed for grafted autogenous bone particles and the newly formed bone (Fig. 5A-H). In clear contrast to these findings, in the biopsy specimens recovered 4 months after conventional bone grafting, markedly few cells were found around the newly formed bone, and ALP-positive signals, albeit weak, were found exclusively on the surface of the bone (Fig. 5I-L). In addition, recruitment of TRAP-positive cells was negligible. These results indicate that the recruitment of both osteoblasts and osteoclasts to the regenerating bone tissue is markedly activated at the site of CAPCs grafting with autogenous bone particle, as compared with that of conventional bone grafting. 

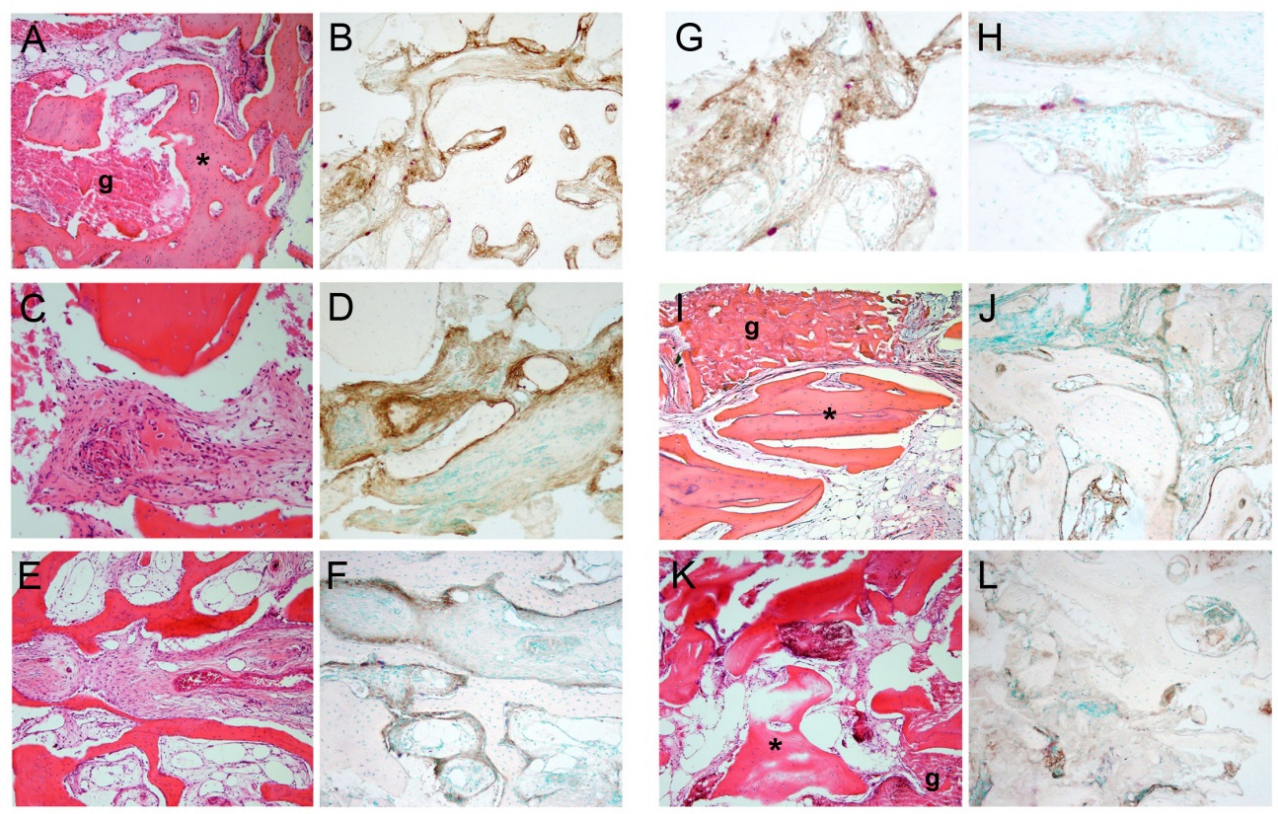

* newly formed bone, g: residual grafted autologous bone particles

Figure 5. Histological analysis of biopsy specimens recovered from the recipient sites at 4 months after bone grafting with CAPCS (A-H) or conventional bone grafting (I-L). A, C, E (H-E staining) and B, D, F, G, H (ALP-TRAP double staining): Active new-bone formation was observed in specimens recovered from the site of a bone graft with CAPCs.

\subsection{CAPC-induced bone remodeling after autogenous bone grafting}

The volume of newly reformed bone tissue at $1 \mathrm{Y}$ was compared to that at $3 \mathrm{M}$ in the cases of maxillary sinus lift with autologous bone and CAPCs and in the cases of maxillary sinus lift with autologous bone only using reconstructed 3D-CT images. Changes in the mean net volume of augmented bone between the two time points were similar regardless of whether CAPCs were used. When compared after categorizing the area of augmented bone according to $\mathrm{CT}$ density, the mean volume was $35 \%$ lower at $1 \mathrm{Y}$ than at $3 \mathrm{M}$ in the area with a CT density $<850 \mathrm{HU}$, regardless of whether CAPCs were used or not (data not shown).

On the other hand, in the high-density area (CT density > 850), marked time-dependent decreases were found only after bone grafting with CAPCs, whereas no decreases were found in conventional bone grafting. In color-coded reconstructed 3D-CT images, high CT density areas believed to correspond to grafted cortical bone particles of mandibular origin contained in the graft material were almost absent in the 3D-CT images taken at $1 \mathrm{Y}$ after autogenous bone grafting with CAPCs, but a smooth surface area with high CT density areas was found on the surface of the augmented bone (Fig. 6). Thus, high-resolution three-dimensional computed tomography (3D-CT) imaging suggested that remodeling of the grafted autogenous 
cortical bone particles was faster in bone grafting with CAPCs than in conventional bone grafting.

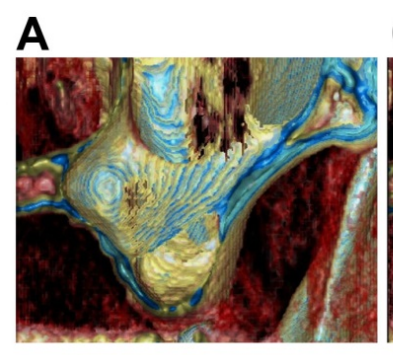

\section{CAPC(+) bone graft}
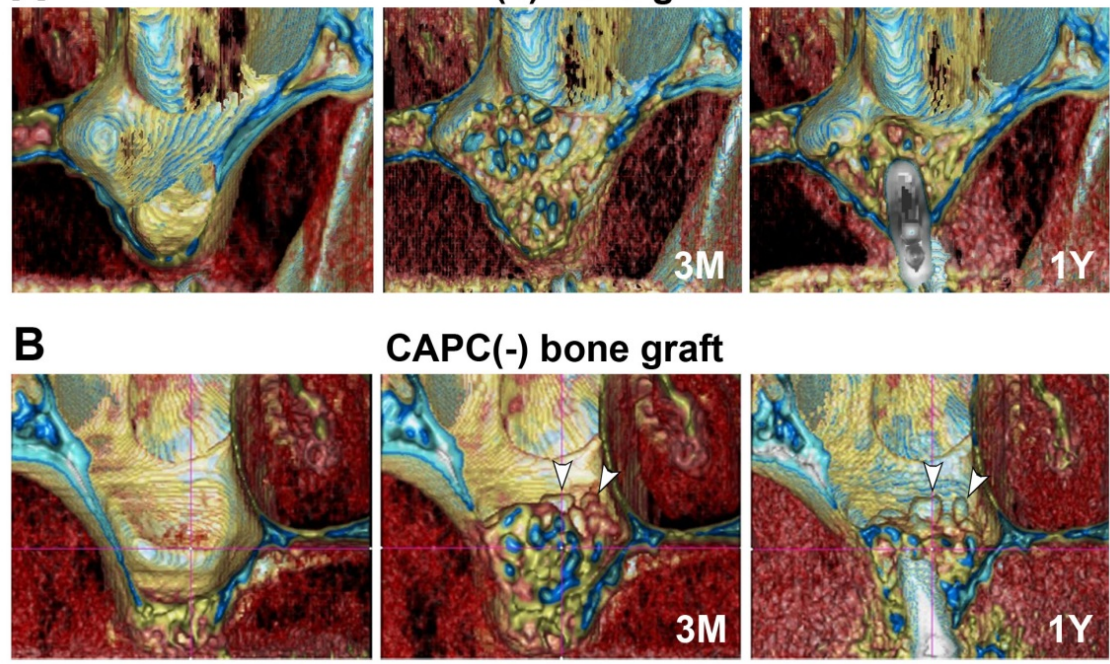

\section{CAPC(-) bone graft}
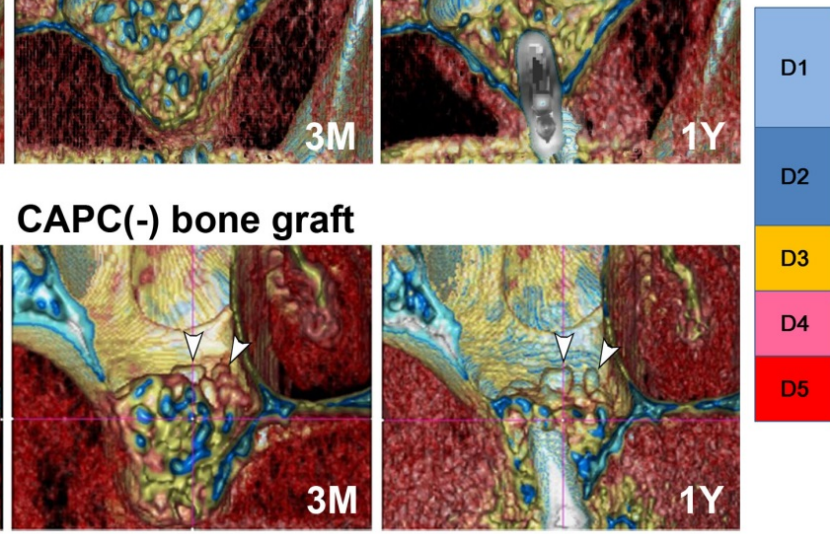

HU

1 1250

Figure 6. CT images of the maxillary sinus constructed from DICOM data before surgery and at 3 months ( $3 \mathrm{M})$ and 1 year (1Y) after CAPC(+) bone graft or conventional CAPC(-) bone grafting. Color mapping of the images according to bone density as determined by Hounsfield units ( $\mathrm{HU})$ for $\mathrm{CT}$ data was applied. In the graft material at 3M, many areas are depicted in light blue (D1) and blue (D2), which is indicative of autogenous cortical bone particles (A and B).

\subsection{Conclusions}

In this clinical research into CAPC use in bone augmentation, we investigated the effects of CAPCs on bone regeneration in patients. Augmentation that is difficult to achieve by conventional autogenous bone grafting, such as that for building bone in both the directions of height and width in the alveolar ridge or for performing a sinus lift of $\geq 15 \mathrm{~mm}$ in patients with a less than 2-mm-thick maxillary sinus floor, are feasible through the application of CAPCs, regardless of age and sex of the patients. The results of histological and 3D-CT analyses suggest that grafted CAPCs effectively recruited osteoblasts and osteoclasts, thereby simultaneously promoting bone formation and remodeling.

The effects of CAPCs in regenerative bone therapy may be exerted via various biological mechanisms, as illustrated in Figure 7. For instance, CAPCs may serve as the original cell population that gives rise to osteoblasts for bone matrix formation and to constituent cells of bone tissue such as vascular endothelial cells, as well as serving as a source of various growth factors, thereby recruiting and activating osteoclasts. 
A

Conventional bone graft

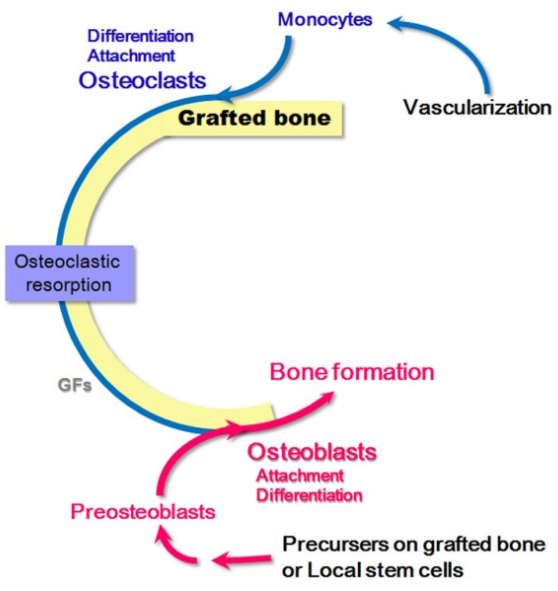

B

\section{Bone graft with cultured autogenous periosteal cells}

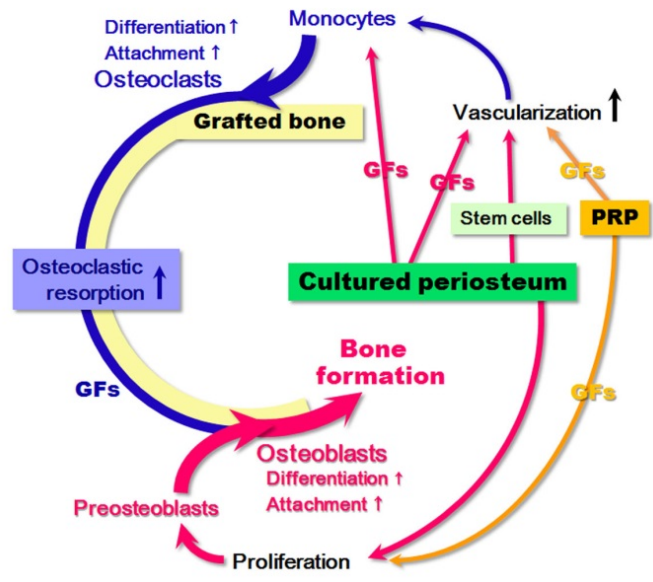

Figure 7. Illustration of hypotheses for bone formation and resorption after (A) conventional bone grafting and (B) after bone grafting with CAPCS.

Bone tissue engineering with CAPC grafting may enable regeneration of bone tissues with complex morphology in a wide area, and thus, the technique will expand the indications for regenerative bone therapy. Currently, the autogenous bone content in the graft material has been reduced to as low as $30 \%$ by use of CAPCs. The less invasive approach reduces the burden on patients and also increases the volume of regenerative bone produced. However, autogenous bone particles serve as a secure scaffold for bone augmentation and thus are not replaceable. The ultimate goal is to establish procedures for CAPC culturing and grafting that enable regenerative bone therapy without harvesting bone. To achieve this goal, we must continue working on developing affordable and effective systems for regenerative bone therapy that also satisfy safety requirements.

\section{Future perspectives of cell-based therapy for periodontal and bone regeneration}

\subsection{Progress in regenerative therapy for periodontium and maxillofacial bones}

First, material-and scaffold-based therapies have been developed using bone replacement graft and GTR membranes. Second, growth factor application has increased for mixed components and recombinant proteins. Several approaches involving enamel-matrix derivatives and PRP/PRF have been reported, and recombinant growth factors (BMP, FGF-2, PDGF, and GDF-5) have been introduced for periodontal and bone regenerative therapy. Third, cellbased therapy has recently emerged using bone marrow cells, periosteal cells, and periodontal 
ligament cells. Finally, combination therapy with scaffolds, growth factors, and cells appears to present a promising strategy to achieve the regeneration of large defects and to enlarge the indication of therapy [Egusa et al., 2012]. Figure 8 shows already used materials and possible candidates in the near future for regeneration of periodontium and maxillofacial bones.

\subsection{Three challenges for combined cell-based therapy}

\subsubsection{Biological and clinical safety challenges}

Prevention of tumor formation following stem cell implantation is a major safety consideration from the biological point of view. It is critical to have an understanding of the cell genetic instability, culture medium and conditions. In addition, practical errors and accidents involving contamination and infection with virus and mycoplasma should be monitored and avoided.

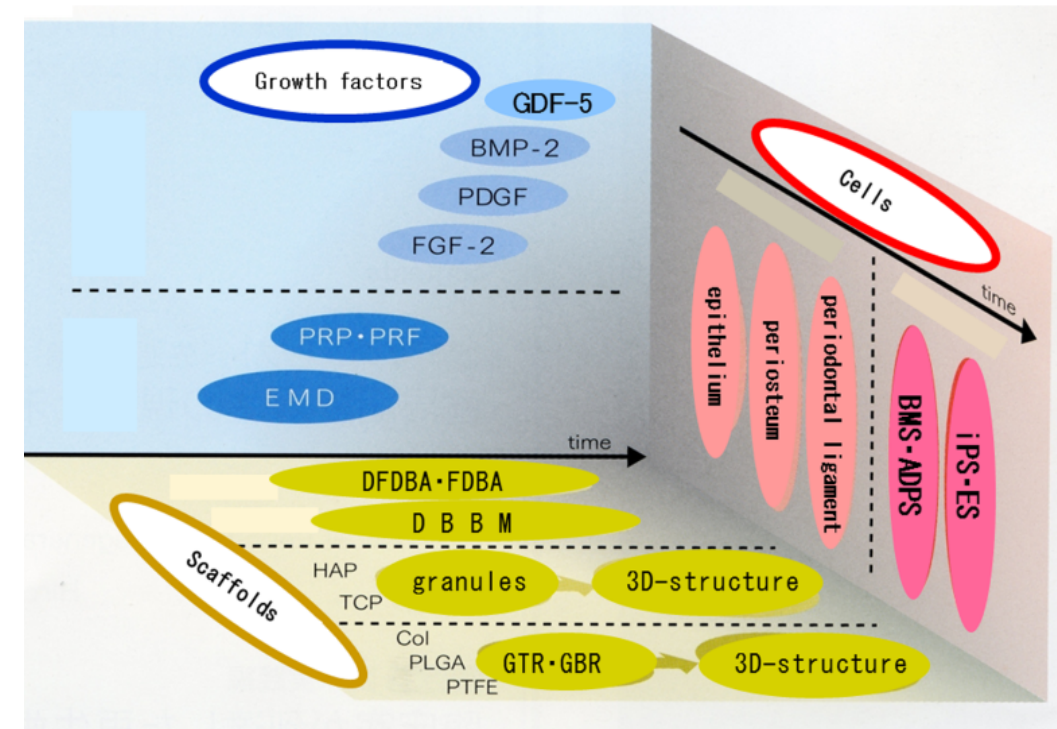

Figure 8. Possible biomaterials, proteins, and cells for regeneration of periodontium and maxillofacial bones.

Growth factor GDF: growth differentiation factor, BMP: bone morphogenetic protein, PDGF: platelet-derived growth factor, FGF: fibroblast growth factor, PRP PRF: platelet-rich plasma platelet-rich fibrin, EMD: enamel matrix derivative

Scaffolds DFDBA: demineralized freeze-dried bone allograft, FDBA: freeze-dried bone allograft, DBBM: demineralized bovine bone matrix, HAP: hydroxyapatite, TCP: tricalcium phosphate, Col: collagen, PLGA: polylactic-polyglycolic acid, PTFE: polytetrafuoroethlene, GTR: guided tissue regeneration, GBR: guided bone regeneration, 3D: three dimensional 
Cells BMS: bone marrow stem cells, ADPS: adipose stem cells, iPS: induced pluripotent stem cell, ES: embryonic stem cells

\subsubsection{Beneficial technical challenges}

It is definitely possible to achieve remarkable regeneration using cell-based therapy compared with conventional regenerative treatment for satisfaction of patients and cost benefit aspects. The matrix and scaffold should have good biocompatibility in terms of the cellular and molecular components during the process of developing and regenerating tissues. The synergistic effects of cell-based therapy with suitable scaffolds and growth factors are anticipated and should be substantiated in the near future.

\subsubsection{Challenges of cell delivery system and cell banking}

Cell processing center/room (CPC) is required for cell-based therapy, and the refinement of techniques to facilitate laboratory handing of cells is also crucial for biological and clinical applications. CPC should be established at hospital, however, it is impossible to establish CPC. Thus, a well-controlled cell delivery system is necessary for wide area of several prefectures. Furthermore, cell banking may be one strategy to realize the potential of cell-based regenerative therapy. Several types of cells can be cryopreserved to retain regenerative potential. Dental cells can be isolated from the cryopreserved tissue whenever required for future regenerative therapies [Chen et al., 2012].

\section{Author details}

Tomoyuki Kawase, Kazuhiro Okuda, Masaki Nagata and Hiromasa Yoshie Institute of Medicine and Dentistry, Niigata University, Japan

\section{References}

[1] Chen FM, Sun HH, Lu H, \& Yu Q. (2012) Stem cell-delivery therapeutics for periodontal tissue regeneration. Biomaterials 33: 6320-6344.

[2] Egusa H, Sonoyama W, Nishimura M, Atsuta I, \& Akiyama K. (2012) Stem cells in dentistry--Part II: Clinical applications. J Prosthodont Res56: 229-248.

[3] Engler AJ, Sen S, Sweeney HL, \& Discher DE. (2006) Matrix elasticity directs stem cell lineage specification. Cell 126: 677-89.

[4] Horimizu M, Kawase T, Tanaka T, Okuda K, Nagata M, Burns DM, \& Yoshie H. (2013) Biomechanical evaluation by AFM of cultured human cell-multilayered periosteal sheets. Micron 48: 1-10. 
[5] Kawase T, Okuda K, Kogami H, Nakayama H, Nagata M, Nakata K, \& Yoshie H. (2009) Characterization of human cultured periosteal sheets expressing bone-forming potential: in vitro and in vivo animal studies. J Tissue Eng Reg Med 3: 218-229.

[6] Kawase T, Okuda K, Kogami H, Nakayama H, Nagata M, \& Yoshie H. (2010a) Osteogenic activity of human periosteal sheets cultured on salmon collagen-coated ePTFE meshes. J Mater Sci Mater Med 21: 731-739.

[7] Kawase T, Okuda K, Kogami H, Nakayama H, Nagata M, Sato T, Wolff LF, \& Yoshie H. (2010b) Human periosteum-derived cells combined with superporous hydroxyapatite blocks used as an osteogenic bone substitute for periodontal regenerative therapy: An animal implantation study using nude mice. J Periodontol 81: 420-427.

[8] Kawase T, Tanaka T, Nishimoto T, Okuda K, Nagata M, Burns DM, \& Yoshie H. (2011) Improved adhesion of human cultured periosteal sheets to a porous poly(Llactic acid) membrane scaffold without the aid of exogenous adhesion biomolecules. J Biomed Mater Res A 98: 100-113.

[9] Kawase T, Uematsu K, Kamiya M, Nagata M, Okuda K, Burns DM, Nakata K, \& Yoshie H. (2014) Real-time quantitative polymerase chain reaction and flow cytometric analyses of cell adhesion molecules expressed in human cell-multilayered periosteal sheets in vitro. Cytotherapy 16: 653-661.

[10] Nagata M, Hoshina H, Li M, Arasawa M, Uematsu K, Ogawa S, Yamada K, Kawase T, Suzuki K, Ogose A, Fuse I, Okuda K, Uoshima K, Nakata K, Yoshie H, Takagi R. (2012) A clinical study of alveolar bone tissue engineering with cultured autogenous periosteal cells: coordinated activation of bone formation and resorption. Bone 50: 1123-1129.

[11] Okuda K, Kawase T, Momose M, Murata M, Saito Y, Suzuki H, Wolff LF, \& Yoshie H. (2003) Platelet-rich plasma contains high levels of platelet-derived growth factor and transforming growth factor- $\$$ and modulates the proliferation of periodontally related cells in vitro. J Periodontol 74: 849-857.

[12] Okuda K, Tai H, Tanabe K, Suzuki H, Sato T, Kawase T, Saito Y, Wolff LF, \& Yoshie H. (2005) Platelet-rich plasma combined with a porous hydroxyapatite graft for the treatment of intrabony periodontal defects in humans: a comparative controlled clinical study. J Periodontol 76: 890-898.

[13] Okuda K, Kawase T, Nagata M, Yamamiya K, Nakata K, Wolff LF, \& Yoshie H. (2009) Treatment of human infrabony periodontal defects by grafting human cultured periosteum sheets combined with platelet-rich plasma and porous hydroxyapatite granules: case series. J Int Acad Periodontol 11: 206-213.

[14] Okuda, K. Kawase, T. Nagata, M. Yamamiya, K. Nakata, K. Wolff, LF. \& Yoshie, H. (2013) Tissue engineered cultured periosteum sheet application to treat infrabony defects: case series and 5-year results. Int J Periodont Rest Dent 33: 281-287. 
[15] Uematsu K, Kawase T, Nagata M, Suzuki K, Okuda K, Yoshie H, Burns DM, \& Takagi R. (2013a) Tissue culture of human alveolar periosteal sheets using a stem-cell culture medium (MesenPRO-RS ${ }^{\mathrm{TM}}$ ): In vitro expansion of CD146-positive cells and concomitant upregulation of osteogenic potential in vivo. Stem Cell Res 10: 1-19.

[16] Uematsu K, Nagata M, Kawase T, Suzuki K, \& Takagi R. (2013b) Application of stem cell media to explant culture of human periosteum: an optimal approach for preparing osteogenic cell material. J Tissue Eng in press.

[17] Vincent LG, Choi YS, Alonso-Latorre B, del Álamo JC, \& Engler AJ. (2013) Mesenchymal stem cell durotaxis depends on substrate stiffness gradient strength. Biotechnol J 8: 472-84.

[18] Yamamiya K, Okuda K, Kawase T, Hata K, Wolff LF, \& Yoshie H. (2008) Tissue-engineered cultured periosteum used with platelet-rich plasma and hydroxyapatite in treating human osseous defects. J Periodontol 79: 811-818. 
